\title{
Deconvolving the Nucleus of Centaurus A Using Chandra PSF Library
}

\author{
Margarita Karovska \\ Harvard-Smithsonian Center for Astrophysics, 60 Garden Street, \\ Cambridge, $M A$
}

T. Aldcroft, M.S. Elvis, I.N. Evans, G. Fabbiano, T.J. Gaetz, T. Isobe, D. Jerius, C. Jones, M. Juda, A. Kenter, D.W. Kim, R.P. Kraft, S.S. Murray, A H. Prestwich, F. Primini, D.A. Schwartz

Harvard-Smithsonian Center for Astrophysics

W. Cui

Purdue University

E.J. Schreier

STSCI

\begin{abstract}
.
We describe preliminary results from our study of multi-scale structures in Centaurus A (NGC 5128) obtained using the Chandra X-ray Observatory HRC-I observations. The high-angular resolution Chandra images reveal X-ray multi-scale structures in this object with unprecedented detail and clarity. The region surrounding the Cen A nucleus, believed to be associated with a supermassive black hole, shows structures on arcsecond scales clearly resolved from the central source.
\end{abstract}

\section{Introduction}

Centaurus A (NGC 5128) is a giant early-type galaxy containing the nearest (at $3.5 \mathrm{Mpc}$ ) radio-bright AGN. Cen A was first imaged in X-rays with Einstein (Feigelson et al 1981), leading to the detection of complex X-ray emission (nucleus, jet, diffuse emission). Later observations with ROSAT led to the detection of emission from the southern inner radio lobe and from a population of point sources (Dobereiner et al 1996). The recently launched Chandra X-ray Observatory provides a unique opportunity for high spatial/spectral resolution studies of the Cen A X-ray emission, including a sub-arcsecond resolution imaging of the nuclear region.

Chandra produces sharper images then any other X-ray telescope to date (less than 0.5 " on axis). The high-spatial resolution is achieved as a result of the innovative design of this observatory including the guidance systems, the mirror assembly (High Resolution Mirror Assembly, or HRMA), and the science 
instruments located in the focal plane. The detectors' pixel sizes and resolution are well matched to capture the sharp images formed by the mirrors and to provide information about the incoming X-rays: their number, position, energy and time of arrival.

In this paper we report preliminary results from our multi-scale spatial analysis of the Chandra High Resolution Camera (HRC-I) observation of Cen A carried out on 1999 September 10 for $17 \mathrm{ksec}$ as part of the Orbital Activation and Calibration phase. The initial analysis and results from this observation are discussed by Kraft et al (1999).

\section{Data Analysis}

We explored the spatial extent of the X-ray sources in the HRC image of Cen A at scales from a fraction of an arcsecond to several arcminutes using two image processing techniques: adaptive smoothing (Ebeling et al 2000), and deconvolution (Richardson 1972 ; Lucy 1974). Crucial to these multi-scale studies is the knowledge of the spatial/spectral characteristics of the HRC Point Spread Functions (PSFs).

The HRMA PSFs vary significantly with source location in the telescope field of view (FOV), as well as with the spectral energy distribution of the source. The HRMA consists of four pairs of nested mirrors (a Wolter Type I design), support structures, and additional thermal and optical baffles system. Because of the Wolter Type I design, the image quality is best in a small area centered about the optical axis. In fact, the mirrors were designed to produce images with better than $1^{\prime \prime}$ resolution; in particular to concentrate better than $85 \%$ of the energy at $0.277 \mathrm{keV}$ within $1^{\prime \prime}$ diameter. This is why a substantial pre- and postlaunch effort was directed at creating a faithful model of the HRMA's mechanical and optical systems. The detailed modeling and metrology of the optics, followed by extensive pre-flight testing and the ongoing on-orbit calibration of the point spread function, showed that we are close to reaching the goal of calibrating the optics' performance to $1 \%$ (Jerius at al 2000).

The simulated Chandra PSFs used in our analysis were generated in two steps; First, ray files are generated using a ray-trace code which models the interaction of photons (rays) passing through the HRMA (Jerius et al 2000). The PSF model images are made by projecting these rays to the detector plane. The pixel size in the model PSFs of $0.13175^{\prime \prime}$ (HRC pixel). Each model PSF was also convolved with a gaussian ( $\sigma$ of $1.5 \mathrm{HRC}$ pixels) in order to simulate the HRC effective resolution (see Kraft et al 2000).

\section{Results}

We performed deconvolution of the full resolution HRC images of the Cen A nucleus with a simulated PSF and with an observed unresolved source (AR Lac) using the Richardson-Lucy technique. The results are similar in both cases, namely using the model PSF and with AR Lac as a point source image, however, we do not have an accurate estimate of the errors. The deconvolved images show that the central source is resolved; The comparison between the sizes of the radially averaged deconvolved images of the Cen A nucleus and that of AR 


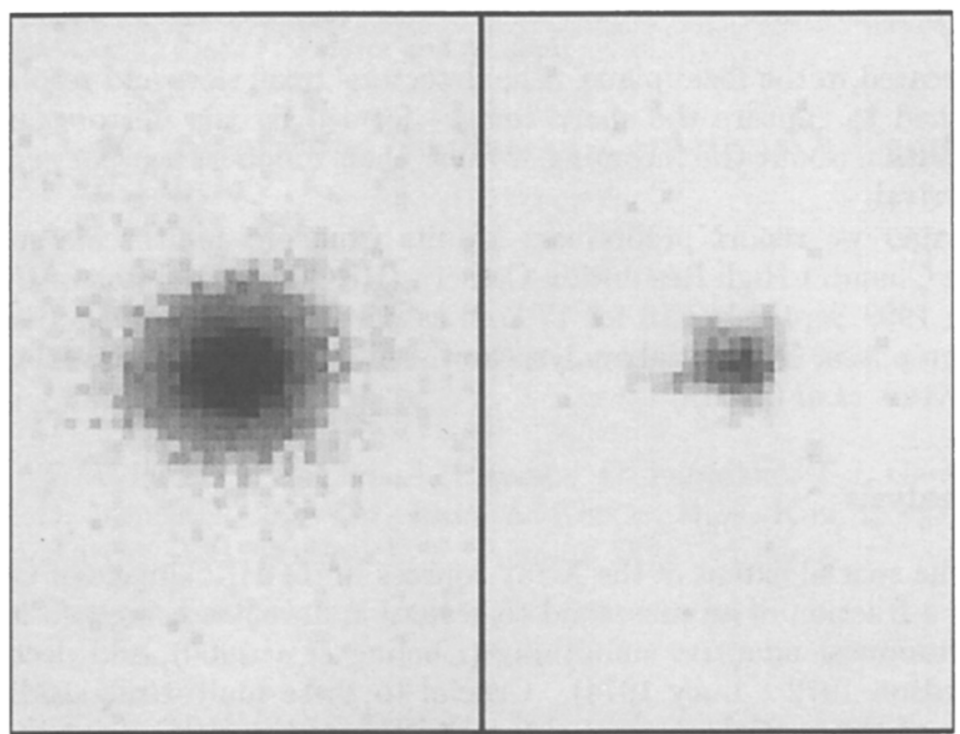

Figure 1. HRC image of Cen A nucleus and the surrounding region ( $\left(5^{\prime \prime} \mathrm{x} 8^{\prime \prime}\right)$ before (left), and after the deconvolution (right). North is up and East is to the left. The pixel size is $0.13175^{\prime \prime}$.

Lac (both deconvolutions were performed using simulated model PSFs) clearly shows that Cen A nucleus is inconsistent with a point source. We estimate its size of approximately $0.3^{\prime \prime}$.

In addition to the bright central source $\left(0.5\right.$ counts $\left.s^{-1}\right)$, the deconvolved image contains several strong asymmetric features (more then an order of magnitude fainter than the nucleus) (Figure 1). We note that the AR Lac image deconvolved with the simulated PSF does not show any of the asymmetries observed in the Cen A image. One of the asymmetric features in the region surrounding the nucleus extends over about $1^{\prime \prime}(20 \mathrm{pc})$ at a position angle of about $35^{\circ}$. It is oriented in the same direction as the extended structure $\left(2^{\prime \prime}\right)$ seen in the HST/NICMOS infrared images of Cen A at a position angle of 33 - (Schreier et al 1998). The orientation of this structure is different from the general orientation of the jet and could be associated with the outer portion of an accretion disk around a black hole (vis Schreier et al 1998). In addition, the HRC image show a nearly perpendicular arc-like structure of a similar size $\left(1-2^{\prime \prime}\right)$, and a possible extension to the South of the nucleus.

We explored the extended emission surrounding the nuclear region at larger scales, from several arcseconds to several arcminutes. The adaptively smoothed images show extended emission within $5^{\prime \prime}$, elongated in the direction of the jet (Figure 2). The model PSF and the AR Lac images smoothed in a similar way did not show any significant asymmetries. It is likely that the extended emission originates from the inner portion of the jet, and could be associated with circumnuclear gas clouds shocked by the X-ray/radio jet. This is supported by the HST/NICMOS observations in Pa $\alpha$ showing two arc-like structures at a distance of about $3^{\prime \prime}$ from the nucleus located at the boundaries of the X-ray emission. At even larger scales, the smoothed images show a low brightness diffuse emission in the direction of the jet. This emission is at least an order 


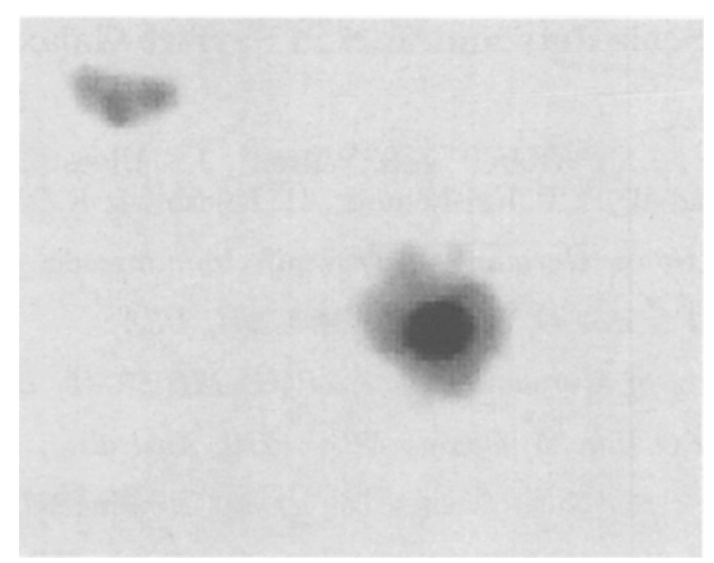

Figure 2. HRC smoothed image of Cen A (20"x $20^{\prime \prime}$ region) containing the nucleus and the emission associated with the A1 knot (upper corner). North is up and East is to the left. For this display, the original HRC image was blocked 4 times and the pixel size is $0.53^{\prime \prime}$.

of magnitude fainter than the extended emission in the vicinity of the nucleus and seems to be connecting the nuclear region with the jet knot A1 located at about $13^{\prime \prime}$ from the nucleus. This is probably emission from the inner portion of the jet which extends continuously from the nuclear region to the bright knots observed further away (Kraft et al 1999).

We also carried out studies of the extended large-scale diffuse emission using the adaptive smoothing images of a $20^{\prime} \times 20^{\prime}$ region centered on the nucleus. The large scale structures suggest complex symmetry, including a component possibly associated with the inner radio lobes (as suggested by the ROSAT HRI data, Dobereiner et al 1996), and a separate component with an orthogonal symmetry that may be associated with the galaxy as a whole.

Acknowledgments. This work was supported by NASA contracts NAS839073 and NAS8-38248

\section{References}

Dobereiner et al 1996, ApJ, 470, L15

Ebeling et al 2000, MNRAS, in press

Feigelson et al 1981, ApJ, 251, 31

Jerius et al 2000, Proc. SPIE 4012

Kraft, R. P et al 2000, ApJ, 531, L9

Lucy, L. B., 1974, AJ, 79, (6), 745

Richardson, W. H., 1972, J. Opt. Soc. Am., 62, (1), 55

Schreier, E. J., et al 1998, ApJ, 499 L143 